\title{
USP18 directly regulates Snail1 protein through ubiquitination pathway in colorectal cancer
}

\author{
Fakun Huang, Chengying Zheng, Longkai Huang, Changqing Lin and Jiaxing Wang ${ }^{*}$ (D)
}

\begin{abstract}
Background: Colorectal cancer (CRC) is one of the most common digestive malignant tumors in the world. Ubiquitin-specific peptidase 18 (USP18) plays a regulatory role in tumorigenesis, and abnormal expression of Snail 1 is also believed to be related to tumorigenesis. However, whether USP18 could affect colorectal cancer through Snail1 remains unclear. This study was designed to investigate the role of USP18 in colorectal cancer.

Methods: USP18 protein and mRNA abundance in clinical tissues and five cell lines were analyzed with quantitative real-time PCR (qRT-PCR) and western blot. USP18 overexpression-treated DLD1 cells and USP18 knockdown-treated SW480 cells were used to study cell proliferation, migration, invasion, and the expression of epithelial-mesenchymal transformation (EMT) biomarkers. Moreover, ubiquitination-related Snail1 degradation was detected with qRT-PCR and western blot. The relationships between USP18 and Snail 1 were investigated with western blot, co-immunoprecipitation, migration, and invasion.
\end{abstract}

Results: USP18 was highly expressed in colorectal cancer tissues. Overexpression of USP18 could promote proliferation, colony formation, migration, and invasion of colorectal cancer cells. Overexpression of USP18 effectively promoted cell survival after treatment with three different chemotherapy drugs. Moreover, USP18 could regulate Snail 1 degradation through ubiquitination pathway. Furthermore, we demonstrated that Snail 1 could effectively reverse the influence of USP18 on cell proliferation, migration, invasion, and EMT of CRC cells.

Conclusion: USP18 could promote the proliferation, migration, and invasion of colorectal cancer by deubiquitinating and stabilizing the Snail1 protein in colorectal cancer.

Keywords: Colorectal cancer, EMT, Snail1, USP18, qRT-PCR

\section{Background}

As one of the most common malignant tumors, colorectal cancer (CRC) remains the third most incident cancer, with the incidence of colon cancer rising rapidly worldwide [1]. Previous data showed that among colorectal cancer patients, $25 \%$ were often accompanied by distant metastases, and about $40-50 \%$ who had not been

*Correspondence: jerry03333@163.com

Department of Gastrointestinal Surgery, The First Affiliated Hospital of Fujian Medical University, 20 Chazhong Road, Fuzhou 350000, Fujian, People's Republic of China found with primary colorectal cancer metastasis eventually developed distant metastasis. Moreover, the median survival of untreated colorectal cancer metastases in advanced patients was only $5-6$ months $[2,3]$. The occurrence and development of colorectal cancer is a process of multi-gene participation and multi-stage evolution. A current research indicates that colorectal cancer is a sequence evolutionary process in which adenomatous polyps eventually become cancerous and metastasize to the primary cancer nest $[4,5]$. The inactivation of tumor suppressor genes (e.g., APC, TP53 and TGFBR2), and the mutation of oncogenes (e.g., RAS, BRAF and PI3KCA), 
which can inhibit or activate downstream-related signaling pathways and ultimately lead to the occurrence of adenomatous polyps and malignant tumors, are involved in the process [6]. Surgical treatment, chemotherapy, radiotherapy and other common clinical and technical methods are difficult to effectively treat late metastatic tumors, which eventually cause $90 \%$ cancer patients death [7]. Therefore, finding new biomarkers and further understanding the molecular mechanism may help prevent and treat colorectal cancer.

Epithelial-mesenchymal transformation (EMT) refers to the transformation of epithelial cells into cells with mesenchymal characteristics [8]. The process includes the loss of epithelial markers such as E-cadherin and cytokeratin, accompanied by increased expression of interstitial markers such as $\mathrm{N}$-cadherin, vimentin and fibronectin [9]. Previous studies suggested that EMT could cause a variety of changes in cells, such as enhanced ability of cell migration and invasion, and enhanced resistance to apoptosis and senescence, which play a very important role in the formation of tumor metastases [10].

Snail1 is considered a key factor in the aggressive expression of tumors for its critical role in the EMT pathway associated with tumor metastasis [11]. Studies showed that Snail1 expression was significantly higher in non-small cell lung cancer tissues than in normal noncancer tissues, which suggested a possible reaction of Snail1 in tumor [12]. Moreover, in normal tissues Snail1 gene was silent, but in tumor tissues its expression was up-regulated, and exerted a functional role by controlling the expression of related proteins [13]. Therefore, it's still worth exploring the detailed molecular mechanism of Snaill in CRC.

Specification peptidase 18 (USP18) is a depolymerase of the ubiquitin-like modified enzyme system that can reduce the modification effect of ISG15 on the target protein by removing ISG15 from the bound target protein, which is called "deubiquitination" and has a regulatory effect on the body's multiple signaling pathways and homeostatic maintainance [14, 15]. Recent studies showed that USP18 could also affect tumorigenesis by regulating interferon production and immune cell function, and recent researches found its expression in various tumors $[16,17]$. The deletion of the USP18 gene induced the expression of exogenous apoptosis-related genes such as TRAIL by activating the I-IFN-related signaling pathway [18]. The knockdown also inhibited the EGFR expression by up-regulating miR-7, which further inhibited the growth of tumor cells and increased apoptosis [19]. Moreover, the lack of USP18 gene could inhibit the formation of leukemia induced by BCR-ABL virus through up-regulating the I-IFN signaling pathway [20]. However, the roles of USP18 and Snaill in CRC are still poorly studied. In this study, we explored whether USP18 affects CRC cells through regulating Snail1 ubiquitination.

\section{Methods \\ Tissue samples and cells}

Sixty colorectal cancer samples and their paired normal tissues were collected in the department of pathology of the First Affiliated Hospital of Fujian Medical University between Jan 2019 and Dec 2019. The ethics committee of The First Affiliated Hospital of Fujian Medical University had reviewed and approved all experimental protocols. All patients had read and signed the informed consent. The detached tissues were quickly frozen with fluid nitrogen and stored at $-80^{\circ} \mathrm{C}$. FHC, HCT116, SW480, DLD1, and LOVO cells were purchased from ATCC (Virginia, USA). Cells were cultured with RPMI 1640 with $10 \%$ FBS (Invitrogen, Carlsbad, CA) in a humidified chamber at 5\% $\mathrm{CO}_{2}$, at $37^{\circ} \mathrm{C}$. SW480 cells were plated on six-well plates $\left(5 \times 10^{5}\right.$ cells per well). OPTI-MEM serum-free medium (M5650, Sigma Aldrich) and Lipofectamine 2000 reagent (Thermo Fisher Scientific, USA) were used in transfection tests. The final concentration of $100 \mathrm{nM}$ siRNA was introduced in this study. Meanwhile, pEZ-Lv201 Vector (Biovector, China) was employed to construct the USP18 overexpression system in the DLD1 cells. Lentiviral particles generated with a standardized protocol were used to produce the highly purified plasmids. Endo FectinLenti $^{\mathrm{TM}}$ and Titer Boost ${ }^{\mathrm{TM}}$ reagents (CWBio, China) were used to co-transfect DLD1 cells. The supernatant was collected after $48 \mathrm{~h}$ transfection and stored at $-80^{\circ} \mathrm{C}$.

\section{Effect of USP18 on chemotherapy sensitivity of CRC cells}

Three common chemotherapy drugs (fluorouracial, doxorubicin, and cisplatin) were used. Overexpression or knockdown of USP18 in CRC cells were established as described above. Then, CRC cells were treated with different concentrations of fluorouracial $(0,20,40,60$, and $80 \mathrm{~g} / \mathrm{mL})$, doxorubicin $(0,0.5,2.5,5$, and $10 \mu \mathrm{M})$, or cisplatin $(0,10,20,30$, and $40 \mu \mathrm{M})$ for $24 \mathrm{~h}$. Then, the cell survival was measured using CCK- 8 assay.

\section{qRT-PCR analysis}

Total RNA was extracted with M5 SuperPure Total RNA Extraction Reagent (SuperTRIgent) (mei5bio, China). The mRNA expression was examined with the Q225 system (Kubotechnology, China). The PCR reaction contained $10 \mu \mathrm{L}$ GoldStar Probe Mixture (CWBio, China), $1 \mu \mathrm{L}$ sense primer $(10 \mathrm{nM}), 1 \mu \mathrm{L}$ anti-sense primer $(10 \mathrm{nM}), 2 \mu \mathrm{L}$ cDNA template $(10 \mathrm{ng})$, and $6 \mu \mathrm{L} \mathrm{H}_{2} \mathrm{O}$. The program for qRT-PCR was set as follows: $95^{\circ} \mathrm{C}, 30 \mathrm{~s}, 40$ cycles $\left(95{ }^{\circ} \mathrm{C}, 5 \mathrm{~s}\right.$, and $\left.60{ }^{\circ} \mathrm{C}, 10 \mathrm{~s}\right) .2^{-\Delta \Delta \mathrm{Ct}}$ cycle method was used to calculate the relative expression level of 
mRNAs. GAPDH was employed as the internal control. Primer sequences used were listed in Additional file 1: Table S1.

\section{Western blot analysis}

Cellular protein in different groups was extracted with 1\% PMSF a RIPA Lysis and Extraction Buffer (Beyotime, China). Sodium dodecy lsulfate-polyacrylamide gel electrophoresis was used to perform further examination. In this step, the proteins were transfered onto a polyvinylidene difluoride layer (Novus, USA). After blocking for $1 \mathrm{~h}$ at room temperature, the layer was brooded with anti-Rabbit USP18 (1:1000) (\#4813, CST, USA), E-cadherin (1:1000) (\# 3195S, CST, USA), Vimentin (1:1000) (\# 5741S, CST, USA), N-cadherin (1:1000) (\#13116S, CST, USA), CD133 (1:1000) (\#64326, CST, USA), CD44(1:1000) (\# 37259S, CST, USA), Snail1 (1:1000) (\#3879, CST, USA), and GAPDH (1:1000) (\#2118, CST, USA), overnight. Proteins were hatched with the corresponding secondary antibodies for $1 \mathrm{~h}$ at room temperature after being treated with ECL Chemiluminescence Detection Kit (PromoCell, German). The bands were observed with Chemiluminescence Imaging (Clinx Ltd., China).

\section{Immunohistochemical staining analysis}

The immunohistochemical SP method was used to stain cancer tissue sections. Tissue sections were baked in a $60{ }^{\circ} \mathrm{C}$ incubator for $1 \mathrm{~h}$, and then were subjected to multiple treatments, including immersion in xylene to dewax, gradient alcohol hydration, microwave antigen repair, and 3\% hydrogen peroxide treatment. After blocking using goat serum, the sections were added in an anti-rabbit USP18 monoclonal antibody Snail1 (1:1000) (\#3879, CST, USA) and incubated at $4{ }^{\circ} \mathrm{C}$ overnight. An optical microscope was used for observation.

\section{Migration and invasion assay}

EZCell $^{\mathrm{TM}}$ Cell Migration/Chemotaxis Assay Kit (24-well) (K911-12, Biovision, USA) and EZCell ${ }^{\mathrm{TM}}$ Cell Invasion Assay (Basement Membrane) (96-well Kit) (K912-100, Biovision, USA) were used to perform cell migration and invasion, respectively.

\section{CCK8}

The differently-treated cells were digested, centrifuged and resuspended. The cells were diluted with complete medium. The cells were counted using a cell glass counting plate, and then diluted to 2000 cells $/ \mathrm{ml} .100 \mu \mathrm{L}$ cell suspension $(2000$ cells $/ \mathrm{mL}$ ) was added to each well in a 96-well plate. There were 5 replicate wells in each group and the five replicates were set and observed at five-time points. Subsequently, we incubated the cells in a $5 \% \mathrm{CO}_{2}$,
$37{ }^{\circ} \mathrm{C}$ incubator overnight. Next day, $10 \mu \mathrm{L}$ CCK-8 solution (Beyotime, China) was added to the medium. Then, the plate was incubated in a $37^{\circ} \mathrm{C}$ incubator for $2 \mathrm{~h}$. The absorbance at OD450 was measured.

\section{Immunofluorescence analysis}

Cancer cells in the logarithmic growth phase were inoculated into 24-well plates with cell slides and cultured for $48 \mathrm{~h}$. We discarded the medium, removed the cell slides, and washed 3 times with PBS. Sections were fixed using $4 \%$ paraformaldehyde at $4^{\circ} \mathrm{C}$ for $30 \mathrm{~min}$. After washing 3 times with PBS (5 min/time), $0.1 \%$ Triton was used to treat sections for $10 \mathrm{~min}$. Then, PBS was used to wash sections for $5 \mathrm{~min}$, and goat serum was used for blocking. After washing 3 times with PBS (5 min/time), first antibody was used to cultivate sections at $4{ }^{\circ} \mathrm{C}$ overnight. Subsequently, secondary antibody was used to culture sections for $1 \mathrm{~h}$ at room temperature in a wet box. After washing 3 times with PBS (10 $\mathrm{min} /$ time), an inverted fluorescence microscope was used to observe results.

\section{Co-IP detection}

Cancer cells in the logarithmic growth phase was used in this step. Total protein was extracted using the RIPA Lysis and Extraction Buffer (89900, ThermolFisher Scientific, USA). The beads were washed with $100 \mu \mathrm{L}$ ice-cold buffer. $100 \mu \mathrm{L}$ antibody binding buffer was added to spin the antibody and magnetic beads for $30 \mathrm{~min}$. The beads were washed 3 times with $200 \mu \mathrm{L}$ buffer. Cell lysate and antibody-conjugated magnetic beads were used to incubate for $1 \mathrm{~h}$ at room temperature and then washed 3 times with $200 \mu \mathrm{L}$ buffer. $20 \mu \mathrm{L}$ elution buffer was used to wash the beads once and the supernatant was collected.

\section{Scratch test}

Differently Snail1 knocked-down cells were resuspended and counted. The scratch test insert after alcohol disinfection was carefully placed in a 12-well plate (3 replicates per group). The complete medium was used to dilute the cells to 500 cells $/ \mu \mathrm{L}$. $70 \mu \mathrm{L}$ cell suspension was added to each well. Twenty-four hours later, the cells were gently washed twice with PBS and then, $1 \mathrm{ml} 1 \%$ FBS medium was added. Cell status was observed under the microscope at $0 \mathrm{~h}$ and $24 \mathrm{~h}$.

\section{Statistical methods}

SPSS16.0 statistical software was used and data were expressed as $\chi \pm s$. Two groups were compared using the $t$ test. One-way analysis of variance was used for comparison between groups. $\mathrm{P}<0.05$ was considered to be significant difference. 


\section{Results}

\section{USP18 gene was highly expressed in CRC tissue}

Sixty CRC patients were included in this study. The clinical features of the 60 patients were shown in the Table 1. The results suggested that significant differences could be calculated in T Stages (I-II) $(\mathrm{P}=0.035)$, Metastasis (N0) $(\mathrm{P}=0.003)$, and Metastasis $(\mathrm{M} 0)(\mathrm{P}=0.025)$. In order to examine the expression of USP1, we first performed the detection in colorectal cancer tissues and the paired normal tissues through online dataset, western blot, qRTPCR, and immunohistochemical staining analysis. For online dataset analysis, UALCAN database (http://ualca n.path.uab.edu/) was applied [21]. The result found that USP18 expression was higher in colorectal cancer tissues than in the paired normal tissues $(\mathrm{P}<0.05)$ (Fig. 1a, b). Meanwhile, western blot analysis revealed that USP18 protein expression was significantly higher in colorectal cancer tissues than in normal tissues (Fig. 1c). qRTPCR analysis indicated that USP18 expression was significantly higher in colorectal cancer tissues than in the paired normal tissues $(\mathrm{P}<0.001)$ (Fig. 1d). Moreover, we analyzed the distribution of the high USP18 expression in colorectal cancer tissues and the paired adjacent tissues. Figure 1e suggested that $80 \%$ (40 of 50) of

\section{Table 1 Clinical features of the patients included in this} study

\begin{tabular}{|c|c|c|c|c|c|}
\hline \multirow[t]{2}{*}{ Features } & \multirow[t]{2}{*}{ Total (n) } & \multicolumn{4}{|l|}{ USP18 } \\
\hline & & Positive & Negative & $x^{2}$ & P-value \\
\hline \multicolumn{6}{|l|}{ Gender } \\
\hline Male & 35 & 29 & 6 & 0.513 & 0.513 \\
\hline Female & 25 & 19 & 6 & & \\
\hline \multicolumn{6}{|l|}{ Age (years) } \\
\hline$\geq 60$ & 38 & 33 & 5 & 3.032 & 0.082 \\
\hline$<60$ & 22 & 15 & 7 & & \\
\hline \multicolumn{6}{|l|}{ T Stages } \\
\hline$|-| \mid$ & 24 & 16 & 8 & 4.444 & $0.035^{*}$ \\
\hline III-IV & 36 & 32 & 4 & & \\
\hline \multicolumn{6}{|l|}{ Metastasis } \\
\hline \multicolumn{6}{|l|}{ N Stages } \\
\hline NO & 15 & 8 & 7 & 8.889 & $0.003^{*}$ \\
\hline $\mathrm{N} 1-2$ & 45 & 40 & 5 & & \\
\hline \multicolumn{6}{|l|}{ M Stages } \\
\hline MO & 45 & 39 & 6 & 5.000 & $0.025^{*}$ \\
\hline M1 & 15 & 9 & 6 & & \\
\hline \multicolumn{6}{|l|}{ Location } \\
\hline Colon & 33 & 25 & 8 & 0.825 & 0.364 \\
\hline Rectal & 27 & 23 & 4 & & \\
\hline \multicolumn{6}{|c|}{ Histological differentiation } \\
\hline Well-moderate & 34 & 27 & 7 & 0.017 & 0.896 \\
\hline Poorly & 26 & 21 & 5 & & \\
\hline
\end{tabular}

high USP18 expression could be detected in colorectal cancer tissues. Furthermore, immunohistochemical staining analysis indicated that USP18 expression was significantly higher in colorectal cancer tissue than in the paired normal tissues $(\mathrm{P}<0.001)$ (Fig. 1f, g). In summary, USP18 expression in colorectal cancer tissues was higher than that in the paired normal tissues.

\section{USP18 promoted proliferation of colorectal cancer cells in vitro}

To further probe the biological function of USP18, we studied USP18 expression in five selected cell lines, FHC, HCT116, SW480, DLD1, and LOVO. Western blot and qRT-PCR analysis of USP18 expression in five cell lines indicated that USP18 protein and mRNA expression were significantly different between each other (Fig. 2a, b). It was notable that USP18 protein and mRNA expression were lower in DLD1 cells than in other cell lines $(\mathrm{P}<0.01)$, and were higher in SW480 cells than in other cell lines $(\mathrm{P}<0.001)$. Therefore, DLD1 and SW480 cells were selected for further study. They were used to construct overexpression and knockdown models of USP18. Figure 2c, d showed that overexpression and knockdown of USP18 in DLD1 and SW480 cells were successfully established. siRNA \#3 and USP18 vector were employed for further study. Meanwhile, we have identified the therapeutic efficiency of overexpression and knockdown in USP18 knockdown-treated SW480 cells, and USP18 overexpression-treated DLD1 cells. Figure 2e, f revealed that overexpression and knockdown system used in this study were both effective. For cell proliferation analysis, USP18 knockdown in SW480 cells could significantly reduce cell proliferation compared to normal SW480 cells on days 2, 3, 4, 5 (Fig. 2g) $(\mathrm{P}<0.01)$. However, USP18 overexpression in DLD1 cells could significantly promote cell proliferation compared to vector-treated DLD1 cells on days 2, 3, 4, 5 (Fig. $2 \mathrm{~h})(\mathrm{P}<0.05)$. Moreover, we further employed edu and CCK-8 experiments to probe the change of cell number in USP18 knocked-down SW480 cells and USP18 over-expressed DLD1 cells. Figure $2 \mathrm{i}, \mathrm{j}$ suggested that similar results of CCK- 8 could be observed in edu experiment. USP18 knockdown in SW480 cells could significantly reduce cell proliferation while USP18 overexpression in DLD1 cells could significantly promote cell proliferation in vitro.

\section{USP18 regulated CRC cell migration and invasion}

In this work, we investigated whether USP18 could regulate colorectal cancer cells migration and invasion in USP18 knockdown-treated SW480 cells and USP18 overexpression-treated DLD1 cells. Figure 3a showed that USP18 knockdown in SW480 cells could significantly inhibit cell scratch ability compared to normal SW480 


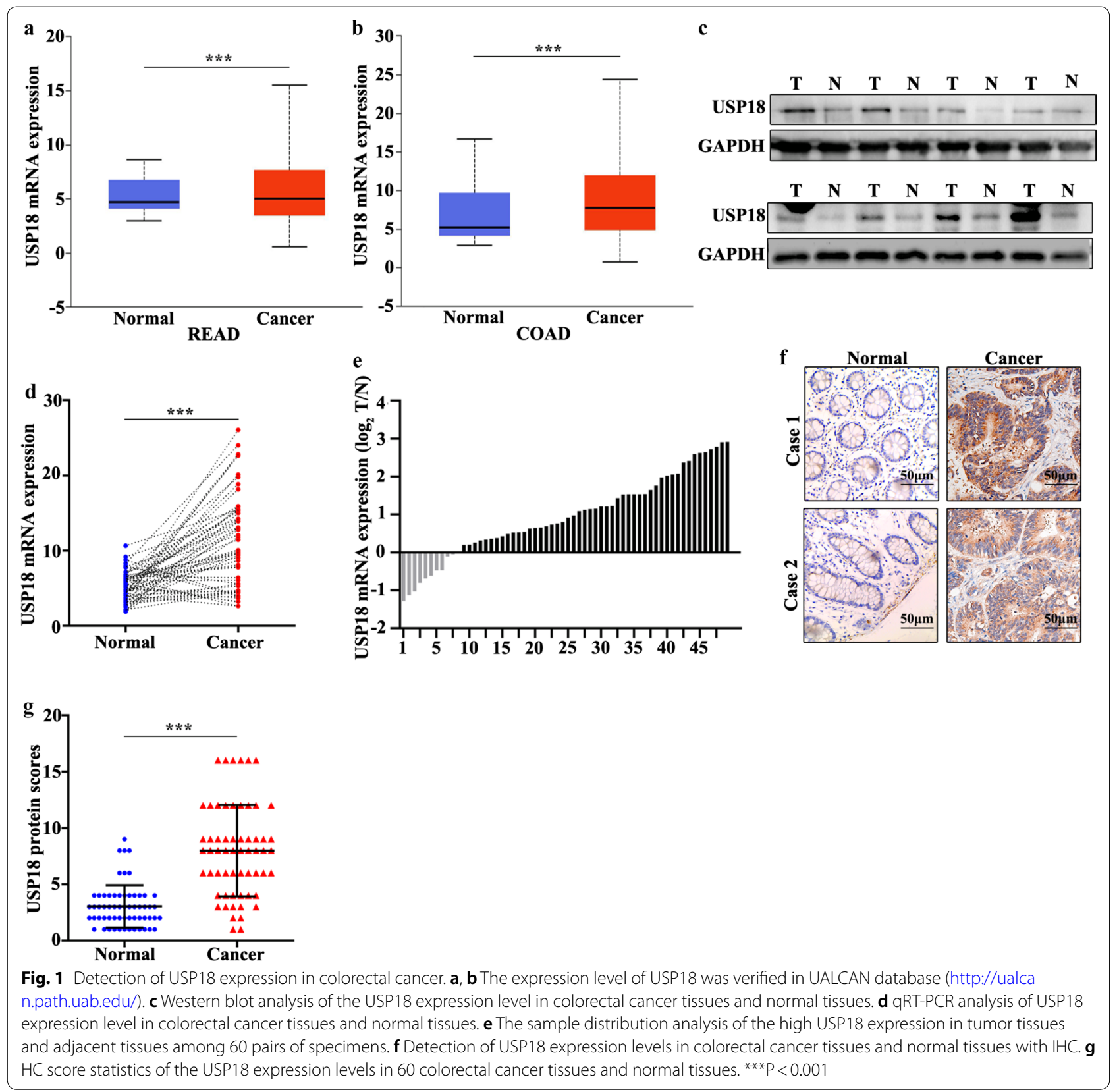

cells $(\mathrm{P}<0.001)$. Meanwhile, USP18 overexpression in DLD1 cells could significantly promote cell scratch ability compared to normal DLD1 cells $(\mathrm{P}<0.01)$ (Fig. 3b). Moreover, we further examined the cell migration and invasion in USP18 knocked-down SW480 cells and USP18 overeexpressed DLD1 cells. USP18 knockdown in SW480 cells could significantly inhibit cell migration and invasion compared with that in the normal SW480 cells $(\mathrm{P}<0.01)$ (Fig. 3c). However, USP18 overexpression in DLD1 cells could promote cell migration and invasion compared with that in the normal DLD1 cells $(\mathrm{P}<0.01)$
(Fig. 3d). Moreover, we further analyzed the protein expression of E-cadherin, N-cadherin and Vimentin. USP18 knockdown in SW480 cells could effectively inhibit the protein expression of $\mathrm{N}$-cadherin and Vimentin $(\mathrm{P}<0.01)$ (Fig. 3e, f), but promote the E-cadherin protein expression $(\mathrm{P}<0.01)$. USP18 overexpression in DLD1 cells could effectively increase the protein expressions of $\mathrm{N}$-cadherin and Vimentin $(\mathrm{P}<0.01)$, but inhibit E-cadherin protein expression $(\mathrm{P}<0.01)$. Therefore, USP18 overexpression in DLD1 cells could promote the protein expression of $\mathrm{N}$-cadherin and Vimentin, but inhibit the 


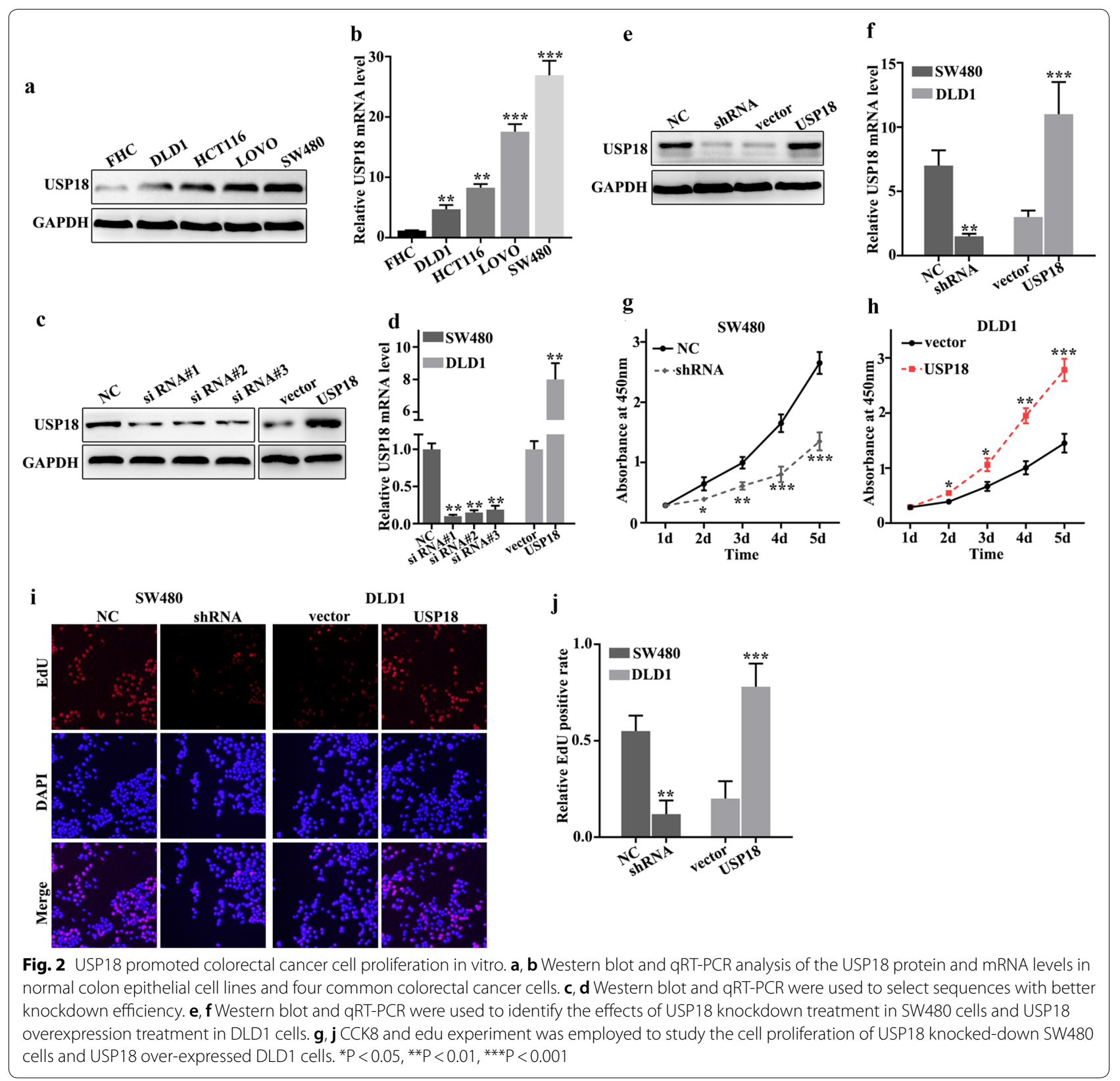

protein expression of E-cadherin. Meanwhile, USP18 knockdown in SW480 cells could inhibit the protein expression of N-cadherin and Vimentin, but promote the protein expression of E-cadherin.

\section{USP18 affected chemotherapy sensitivity of CRC cells}

We investigated whether the changes of USP18 expression could affect the chemotherapy sensitivity of colorectal cancer cells using three common chemotherapeutic molecular drugs, including fluorouracial, doxorubicin and cisplatin Different concentrations of fluorouracial $(0,20,40,60$, and $80 \mathrm{~g} / \mathrm{mL})$, doxorubicin $(0,0.5,2.5,5$, and $10 \mu \mathrm{M})$, and cisplatin $(0,10,20,30$, and $40 \mu \mathrm{M})$ were applied in this study. The results suggested that USP18 knockdown in SW480 cells could significantly decrease cell survival in three different drug treatments compared to normal SW480 cells $(\mathrm{P}<0.05)$ (Fig. $4 \mathrm{a}-\mathrm{c}$ ). However, USP18 overexpression in DLD1 cells could effectively promote cell survival in three different drug treatments compared to normal DLD1 cells $(P<0.05)$ (Fig. $4 \mathrm{~d}-\mathrm{f}$ ). These results revealed that USP18 knockdown in SW480 cells and USP18 overexpression 


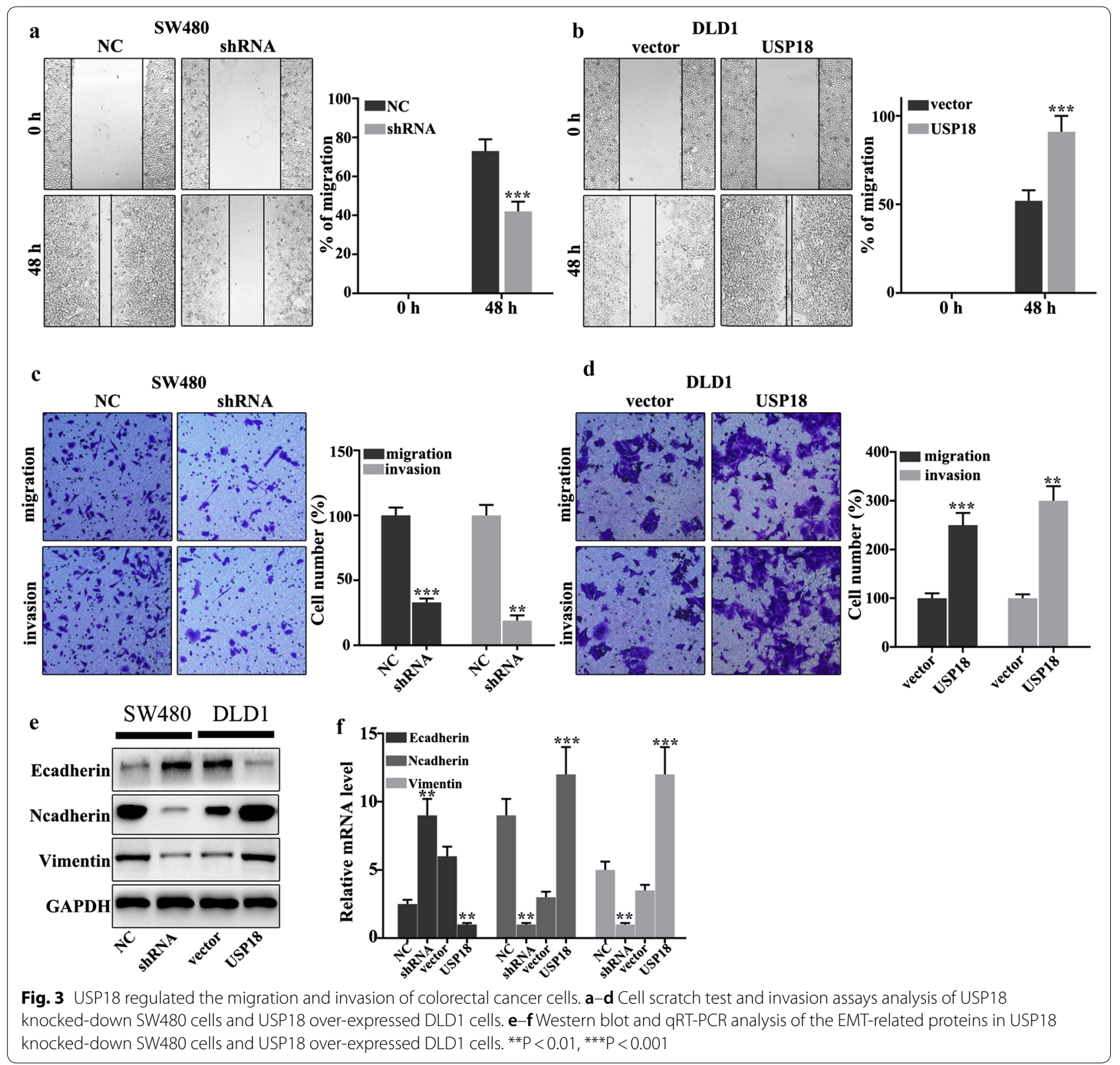

in DLD1 cells were closely related to cell survival rate changes caused by drug treatments. Moreover, we evaluated the protein and mRNA expression of stem cell-related biomarkers (i.e., CD44 and CD133). It is indicated that USP18 knockdown in SW480 cells could significantly decrease protein and mRNA expression of stem cell-related biomarkers, while USP18 overexpression in DLD1 cells could effectively promote protein and mRNA expression of stem cell-related biomarkers $(\mathrm{P}<0.001)$ (Fig. 4g, h). The above results suggested that USP18 expression was closely related to stem cell ability.
USP18 regulated Snail1 expression through ubiquitination In this study, we further studied the potential relationships between the expressions of USP18 and Snail1. We further analyzed the potential relationships of the mRNA expressions of USP18 and Snail1 in 60 clinical samples (Fig. 5a, b). The results suggested that no correlation could be observed between the mRNA expressions of USP18 and Snail1 in clinical samples. However, a significant correlation could be observed between the protein expressions of USP18 and Snail1 $(\mathrm{P}=0.000)$. Moreover, western blot and qRT-PCR were employed to investigate the protein and mRNA expressions of Snail1 


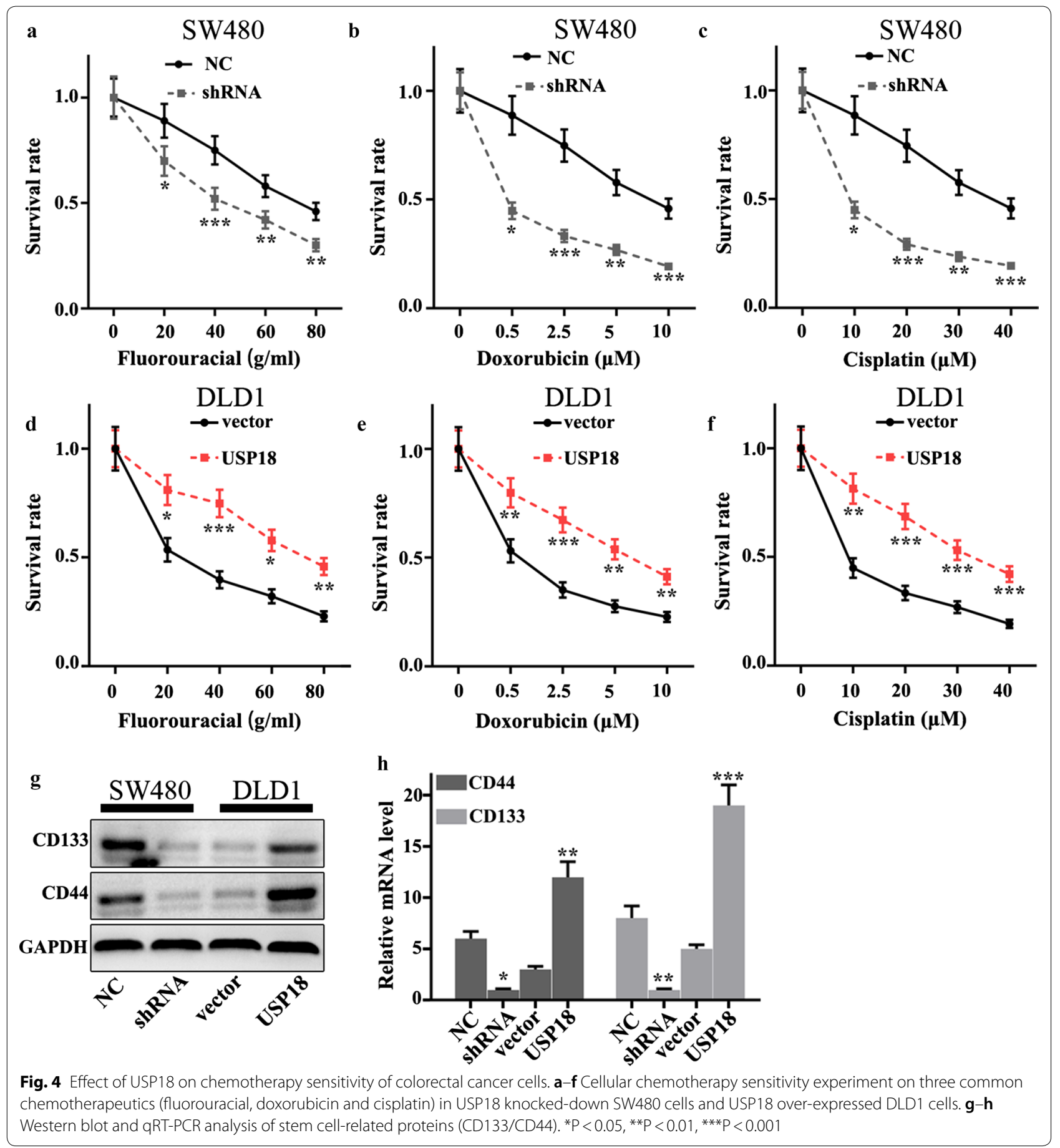

in USP18 knocked-down SW480 cells, and USP18 overexpressed DLD1 cells. Figure $5 \mathrm{c}$ showed that Snail1 protein expression was lower in USP18 knocked-down SW480 cells than in normal SW480 cells, but Snail1 protein expression was more abundant in USP18 overexpressed DLD1 cells than in normal DLD1. It was notable that no difference of Snail1 mRNA abundance could be detected in USP18 knocked-down SW480 cells and USP18 over-expressed DLD1 cells (Fig. 5d). The above results suggested that USP18 could affect the Snail1 protein expression but not Snail1 mRNA level. Moreover, we investigated the potential relationships between USP18 ubiquitination and Sanil1 in USP18 over-expressed DLD1 cells. MG132 is the inhibitor of proteasome 

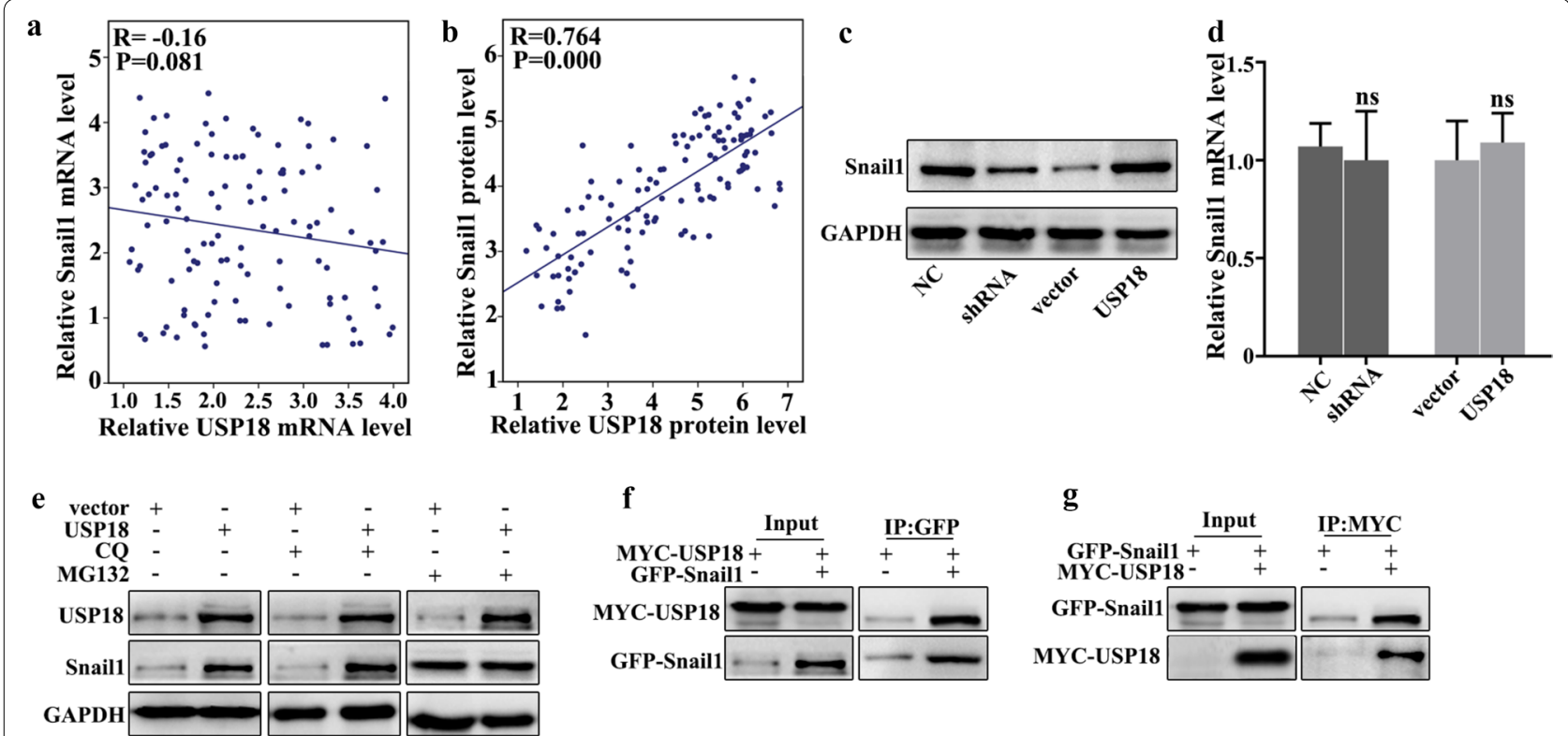
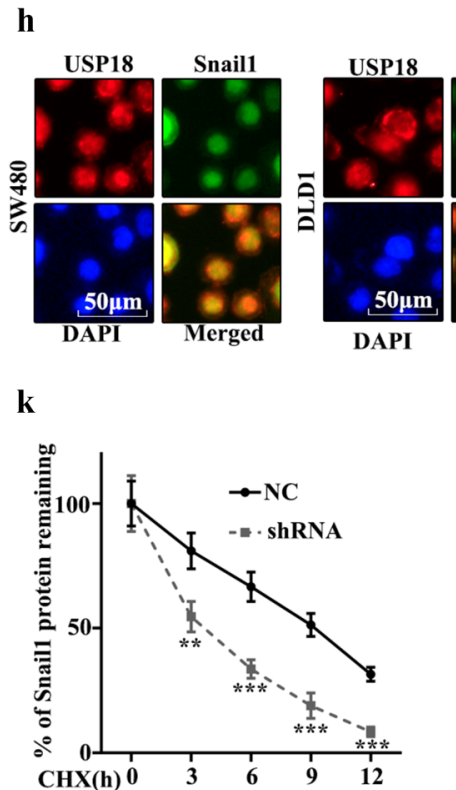
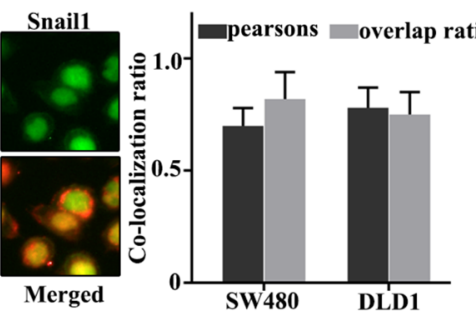

I
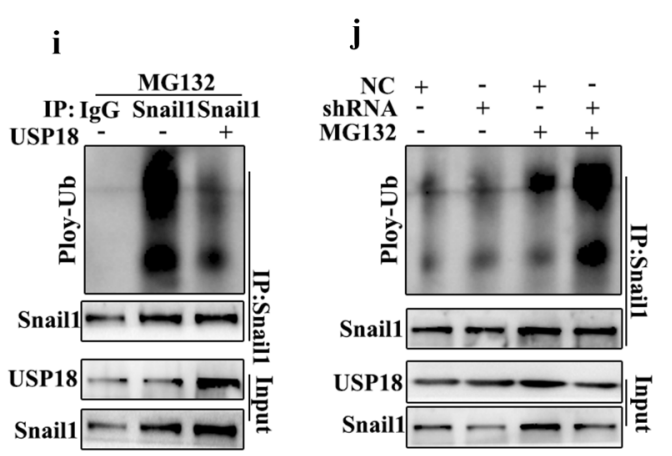

Fig. $\mathbf{5}$ USP18 regulated Snail1 expression through ubiquitination. a Correlation verification between USP18 and Snail1 mRNA in 60 colon cancer specimens. b The correlation between USP18 and Snail1 protein expression was calculated in 60 pairs of colon cancer specimens. c- d Western blot and qRT-PCR analysis the relationships between USP18 and Snail1. e Snail1 protein expression analysis with CQ and MG132 treatments. $\mathbf{f}$ Co-precipitation analysis of USP18 and Snail1 protein in SW480 cell. g Co-localization of immunofluorescence analysis of USP18 and Snail1 in SW480 and DLD1 cell. $\mathbf{h}$ Co-precipitation of USP18 and Snail 1 in the 293 T cell. $\mathbf{i}$ Ubiquitination testing of USP18 in DLD1 cell line. $\mathbf{j}$ Ubiquitination testing of USP18 in SW480 cell line. k, I Cycloheximide (protein degradation rate) analysis showed that USP18 could inhibit Snail1 protein degradation. ${ }^{*} \mathrm{P}<0.05,{ }^{* *} \mathrm{P}<0.01,{ }^{* * *} \mathrm{P}<0.001$

degradation pathway in the cell and Chloroquine (CQ) is an inhibitor of autophagolysosomal degradation pathway. Therefore, we employed MG132 and CQ to study the protein expressions of USP18 and Snail1 in USP18 overexpressed DLD1 cells (Fig. 5e). The results suggested that
MG132 could effectively increase Snail1 protein expression but CQ exerted no effect. Therefore, we speculated that USP18 could regulate Snail1 expression through the ubiquitination but not the cellular autophagy pathway. Moreover, we examined the potential interaction 
between USP18 protein and Snail1 protein in cellular using Co-immunoprecipitation (Co-IP). Figure $5 \mathrm{f}$ showed that USP18 protein could interact with Snail protein. Moreover, we carried out the forward and reverse protein Co-IP of USP18 protein and Snail1 protein in USP18 over-expressed DLD1 cells. Figure $5 \mathrm{~g}$ further identified the interaction between USP18 protein and Snail1 protein. Furthermore, immunofluorescence colocalization analysis of USP18 protein and Snaill revealed that the spatial distribution of both molecular was overlapping. The above result further illustrated the mutual combination between USP18 protein and Snail1 in USP18 over-expressed DLD1 cells (Fig. 5h). Furthermore, we employed ubiquitin detection in USP18 knocked-down SW480 cells and USP18 over-expressed DLD1 cells (Fig. 5i, j). The results suggested that USP18 knockeddown SW480 cells could significantly promote the degradation of the remaining Snail1 protein in cells compared to that in normal SW480 cells $(\mathrm{P}<0.01)$. Meanwhile, USP18 over-expressed DLD1 cells could significantly decrease the degradation of the remaining Snail1 protein in cells compared to that in normal DLD1 cells $(\mathrm{P}<0.01)$. Furthermore, we have further employed cycloheximide (CHX) to study the relationships between protein expressions of USP18 and Snail in USP18 knocked-down SW480 cells and USP18 over-expressed DLD1 cells. Figure $5 \mathrm{k}, 1$ suggested that USP18 over-expressed DLD1 cells could significantly promote the remaining Snail1 protein in cells compared to normal DLD1 cells $(P<0.01)$. Meanwhile, USP18 knocked-down SW480 cells could significantly decrease the remaining Snail1 protein in cells compared to normal SW480 cells $(\mathrm{P}<0.01)$.

\section{Snail1 could effectively reverse the influence of USP18 on cell proliferation, migration, invasion, and EMT of CRC cells}

In this study, we further performed the rescue experiment to demonstrate the relationship between USP18 and Snail1. We introduced Snail1 knockdown and overexpression treatment in USP18 knockdown-treated SW480 cells and USP18 overexpression-treated DLD1 cells. Figure 6a, b showed that siSnail1 \#3 and Snail1 vector were effective to establish knockdown and overexpression models of Snail1. Meanwhile, Fig. 6c, d suggested that knockdown and overexpression treatment of Snail1 gene treatments could effectively inhibit and promote cell proliferation caused by USP18 overexpression and knockdown treatments, respectively. Moreover, we studied the cell scratch of the Snaill knockdown and overexpression treatments in DLD1 cells, which had been treated with USP18 overexpression and USP18 knockdown, respectively. Figure 6e showed that the Snail1 knockdown could effectively reverse the changes in cell scratch caused by USP18 knockdown $(\mathrm{P}<0.01)$. Meanwhile, Snail1 overexpression could effectively reverse the changes of invasion caused by USP18 overexpression in SW480 cells $(\mathrm{P}<0.01)$ (Fig. 6f). Furthermore, we studied the effect of USP18 and Snail1 on chemotherapy sensitivity of colorectal cancer. Figure $6 \mathrm{~g}$, h suggested that Snail1 overexpression and knockdown treatments could effectively reverse the changes in cell survival rate caused by USP18 knockdown and overexpression treatment in the chemotherapy sensitivity analysis $(P<0.01)$. In summary, knockdown and overexpression of Snail1 could effectively reverse the influence of USP18 on cell proliferation, migration, invasion, and EMT.

\section{Discussion}

In China, colorectal cancer remains as one of the most common malignant tumors in the digestive system, with a third incidence rate among all tumors and being increasing over the past decades due to environmental and dietary factors [22, 23]. Studies have found the relationship between its incidence and intestinal polyposis, chronic stress and inflammation, and a family history of cancer [24]. Previous studies suggested that the main cause of death was the invasion and migration of advanced colorectal cancer [25]. Improving diagnostic techniques, including surgery, chemotherapy and radiotherapy treatments, could help detect tumors early and improve patient survival. Better understanding the pathogenesis progression may provide new therapeutic strategies for the prevention and treatment of CRC.

USP18 is an effective regulator of epidermal growth factor receptors (EGFR) [19], and the low expression of it could lead to a down-regulated expression of carcinogenic targets thus decreasing cell proliferation and increasing cell apoptosis [26]. In MCF-7 cells and glioblastoma, knocking out USP18 can induce apoptosis of tumor cells $[27,28]$. The low expression of USP18 can significantly reduce the metastasis and invasion of lung cancer cells [29]. However, what role USP18 plays in the progression of CRC has not been reported.

Ubiquitination plays an important role in several biological processes including metabolism, protein degradation, cellular localization, inflammatory immunity, transcription regulation, and cell cycle [30]. Meanwhile, ubiquitination is closely linked with the regulation of tumors. It was reported that over-expressed DUSP4 could promote chemotherapy-induced apoptosis. In addition, silence of DUSP4 could activate the Ras-ERK signaling pathway and further promote the proliferation and migration of tumor cells [31]. However, the role of USP18 in the regulation of tumor cells is poorly understanded. In this study, we demonstrated that the change of USP18 expression was closely related to the invasion, 


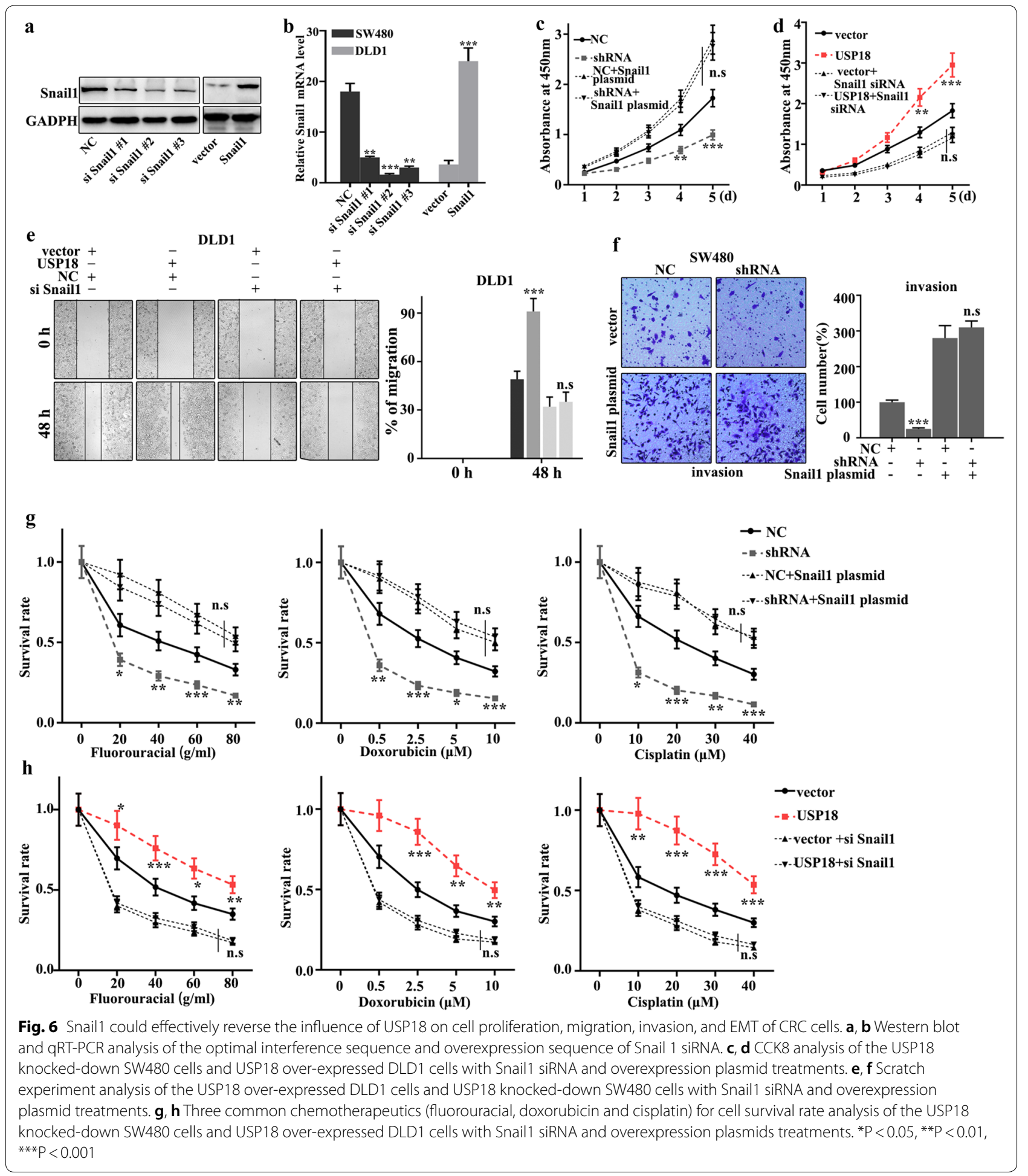

migration, and proliferation of CRC cells. Therefore, USP18 may provide a potential target for the treatment of CRC. Recent reports indicated that abnormal expression of USP18 in CRC tissues was associated with a poorer prognosis [17]. Those results are consistent with our findings, which showed that the expression of USP18 was higher in CRC tissues than in adjacent tissues, and overexpression or knockout of USP18 could affect the 
proliferation, and migration of CRC cells. Therefore, USP18 might be a biomarker for the diagnosis of CRC.

Snail1 is a nuclear transcription factor that can control the transcription efficiency of DNA to messenger RNA [32]. Previous study suggested that Snaill can inhibit the expression of the downstream gene Cyclin D2 and promote cell survival [33]. Meanwhile, it was suggested that Snail1 can upregulate the expression of myosin that could promote the migration of tumor cells [34]. However, whether USP18 could affect the proliferation, migration and invasion of CRC cells through targeting Snail1 remains unclear.

EMT process has been believed to act a key role affecting tumor metastasis. EMT is characterized by decreased expression of epithelial proteins such as E-Cadherin, and increased level of mesenchymal protein such as vimentin. Low E-Cadherin expression means decreased of epithelial connexin, and further facilitate tumor metastasis. In this study, we found that E-Cadherin, Neadherin, and Vimentin were remarkably influenced in the overexpression and knockdown models of USP18. Protein stability is mainly affected by proteasome degradation pathways and autophagolysosomal degradation pathways.

Snail1 is an important transcription factor of EMT, and the expression level of it is linked with the invasion, migration, and apoptosis of tumor cells. Snail1 is believed to be an important factor affecting the neural tube and development of mesoderm, but also plays an important role in tumor metastasis. Snaill is the most important E-cadherin transcriptional repressor, and it could downregulate the expression of claudins and occludins protein. Our results revealed that DUSP18 and Snail1 could regulate EMT of CRC through E-caderin, $\mathrm{N}$-caderin, and Vitmentin. Snail1 can directly interact with USP18 in cellular.

In this study, it was notable that Snaill expression was significantly affected in USP18 over-expressed or knocked-down cells. Snaill could directly interact with USP18 in cells. Moreover, USP18 could reduce Snail1 protein expression without affecting its transcription. Moreover, our results suggested that USP18 affected the protein degradation pathway of Snail1 through ubiquitination modification. Meanwhile, knockdown and overexpression of Snail1 could affect cell migration and invasion, and EMT-related molecules including E-cadherin, N-cadherin, and Vimentin. We proved that Snail1 could effectively reverse the influence of USP18 on cell proliferation, migration, invasion, and EMT of CRC cells.

\section{Conclusions}

We demonstrated that high USP18 expression could be detected in colorectal cancer tissues and cells. USP18 might regulate the cell proliferation, invasion, migration, and EMT process of CRC cells through targeting Snail1 ubiquitylation degradation pathway. This might provide a novel thought for the prevention and treatment of CRC by targeting USP18/Snail1.

\section{Supplementary information}

Supplementary information accompanies this paper at https://doi. org/10.1186/s12935-020-01442-1.

Additional file 1. Sequence of primers for Quantitative reverse transcription-PCR.

Abbreviations

CRC: Colorectal cancer; USP18: Ubiquitin-specific peptidase 18; qRT-PCR: Quantitative real-time PCR test; EMT: Epithelial-mesenchymal transformation; CQ: Chloroquine; Co-IP: Co-immunoprecipitation; CHX: Cycloheximide; EGFR: Epidermal growth factor receptors.

\section{Acknowledgements}

None.

\section{Authors' contributions}

$\mathrm{FH}$ and JW conceived and designed the project; CZ and LH collected the data; $\mathrm{CZ}$ and $\mathrm{CL}$ performed the interpretation of data and statistical analysis; $\mathrm{FH}$ wrote the manuscript; JW revised the paper. All authors read and approved the final manuscript.

Funding

None.

\section{Availability of data and materials}

All data generated or analysed during this study are included in this published article.

\section{Ethics approval and consent to participate}

The ethics committee of The First Affiliated Hospital of Fujian Medical University had reviewed and approved all experimental protocols. All patients had read and signed the informed consent.

\section{Consent for publication}

Not applicable.

\section{Competing interests}

The authors declare that they have no competing interests.

Received: 22 May 2020 Accepted: 20 July 2020

Published online: 28 July 2020

\section{References}

1. Siegel RL, Miller KD, Jemal A. Cancer statistics, 2019. CA Cancer J Clin. 2019;69(1):7-34. https://doi.org/10.3322/caac.21551.

2. Valderrama-Treviño Al, Barrera-Mera B, Ceballos-Villalva JC, Montalvo-Javé EE. Hepatic Metastasis from Colorectal Cancer. Eur J Hepato-gastroenter. 2017;7(2):166-75. https://doi.org/10.5005/jp-journals-10018-1241.

3. Vatandoust S, Price TJ, Karapetis CS. Colorectal cancer: metastases to a single organ. World J Gastroenterol. 2015;21(41):11767-76. https://doi. org/10.3748/wjg.v21.i41.11767.

4. Simon K. Colorectal cancer development and advances in screening. Clin Interv Aging. 2016;11:967-76. https://doi.org/10.2147/cia.s109285.

5. Sievers CK, Grady WM, Halberg RB, Pickhardt PJ. New insights into the earliest stages of colorectal tumorigenesis. Exp Rev Gastroenterol Hepatol. 2017;11(8):723-9. https://doi.org/10.1080/17474124.2017.1330150. 
6. Nguyen $\mathrm{HT}$, Duong $\mathrm{HQ}$. The molecular characteristics of colorectal cancer: implications for diagnosis and therapy. Oncol Lett. 2018;16(1):9-18. https://doi.org/10.3892/ol.2018.8679.

7. Hong SN. Genetic and epigenetic alterations of colorectal cancer. Intestinal Res. 2018;16(3):327-37. https://doi.org/10.5217/ir.2018.16.3.327.

8. Das V, Bhattacharya S, Chikkaputtaiah C, Hazra S, Pal M. The basics of epithelial-mesenchymal transition (EMT): a study from a structure, dynamics, and functional perspective. J Cell Physiol. 2019. https://doi.org/10.1002/ jcp.28160.

9. Lamouille S, Xu J, Derynck R. Molecular mechanisms of epithelial-mesenchymal transition. Nat Rev Mol Cell Biol. 2014;15(3):178-96. https://doi. org/10.1038/nrm3758.

10. Roche J. The epithelial-to-mesenchymal transition in cancer. Cancers. 2018;10:2. https://doi.org/10.3390/cancers10020052.

11. Szynglarewicz B, Kasprzak P, Donizy P, Biecek P, Halon A, Matkowski R. Biological aggressiveness of subclinical no-mass ductal carcinoma in situ (DCIS) can be reflected by the expression profiles of epithelial-mesenchymal transition triggers. Int J Mol Sci. 2018;19:12. https://doi.org/10.3390/ ijms19123941.

12. Liu CW, Li CH, Peng YJ, et al. Snail regulates Nanog status during the epithelial-mesenchymal transition via the Smad1/Akt/GSK3ß signaling pathway in non-small-cell lung cancer. Oncotarget. 2014;5(11):3880-94. https://doi.org/10.18632/oncotarget.2006.

13. Wu Y, Zhou BP. Snail: more than EMT. Cell Adhes Migr. 2010;4(2):199-203. https://doi.org/10.4161/cam.4.2.10943.

14. Zhang D, Zhang DE. Interferon-stimulated gene 15 and the protein ISGylation system. J Interf Cytok Res. 2011;31(1):119-30. https://doi. org/10.1089/jir.2010.0110

15. Jiménez Fernández D, Hess S, Knobeloch KP. Strategies to target ISG15 and USP18 toward therapeutic applications. Front Chem. 2019;7:923. https://doi.org/10.3389/fchem.2019.00923.

16. Budhwani M, Mazzieri R, Dolcetti R. Plasticity of type I interferon-mediated responses in cancer therapy: from anti-tumor immunity to resistance. Front Oncol. 2018;8:322. https://doi.org/10.3389/fonc.2018.00322.

17. Zuo C, Sheng X, Ma M, Xia M, Ouyang L. ISG15 in the tumorigenesis and treatment of cancer: an emerging role in malignancies of the digestive system. Oncotarget. 2016;7(45):74393-409. https://doi.org/10.18632/ oncotarget.11911.

18. Honke N, Shaabani N, Zhang DE, Hardt C, Lang KS. Multiple functions of USP18. Cell Death Dis. 2016;7(11):e2444. https://doi.org/10.1038/cddis .2016 .326$.

19. Duex JE, Comeau L, Sorkin A, Purow B, Kefas B. Usp18 regulates epidermal growth factor (EGF) receptor expression and cancer cell survival via microRNA-7. J Biol Chem. 2011;286(28):25377-86. https://doi. org/10.1074/jbc.M111.222760

20. Yan M, Luo JK, Ritchie KJ, et al. Ubp43 regulates BCR-ABL leukemogenesis via the type 1 interferon receptor signaling. Blood. 2007;1 10(1):305-12. https://doi.org/10.1182/blood-2006-07-033209.

21. Chandrashekar DS, Bashel B, Balasubramanya SAH, et al. UALCAN: a portal for facilitating tumor subgroup gene expression and survival analyses.
Neoplasia (New York, NY). 2017;19(8):649-58. https://doi.org/10.1016/j. neo.2017.05.002.

22. Wen $\mathrm{D}$, Zou $\mathrm{W}$, Wen $\mathrm{X}$, et al. Urban-rural disparity in colorectal cancer incidence and increasing trend in relation to socioeconomic development and urbanization in China. J Int Med Res. 2018:46(10):4181-96. https:// doi.org/10.1177/0300060518791090.

23. Pourhoseingholi MA, Vahedi M, Baghestani AR. Burden of gastrointestinal cancer in Asia; an overview. Gastroenterol Hepatol Bed Bench. 2015;8(1):19-27.

24. Jasperson KW, Tuohy TM, Neklason DW, Burt RW. Hereditary and familial colon cancer. Gastroenterology. 2010;138(6):2044-58. https://doi. org/10.1053/j.gastro.2010.01.054.

25. Mármol I, Sánchez-de-Diego C, Pradilla Dieste A, Cerrada E, Rodriguez Yoldi MJ. Colorectal Carcinoma: a General Overview and Future Perspectives in Colorectal Cancer. Int J Mol Sci. 2017;18:1. https://doi. org/10.3390/ijms18010197.

26. Potu H, Sgorbissa A, Brancolini C. Identification of USP18 as an important regulator of the susceptibility to IFN-alpha and drug-induced apoptosis. Cancer Res. 2010;70(2):655-65. https://doi.org/10.1158/0008-5472. can-09-1942.

27. Xu P, Duong DM, Seyfried NT, et al. Quantitative proteomics reveals the function of unconventional ubiquitin chains in proteasomal degradation. Cell. 2009;137(1):133-45. https://doi.org/10.1016/j.cell.2009.01.041.

28. Chou CK, Chang YT, Korinek M, et al. Correction: Chon-Kit Chou, et al. The Regulations of Deubiquitinase USP15 and Its Pathophysiological Mechanisms in Diseases. Int. J. Mol. Sci. 2017, 18, 483. International journal of molecular sciences. 2017; 18: 5. https://doi.org/10.3390/ijms18050902.

29. Rahighi S, Ikeda F, Kawasaki M, et al. Specific recognition of linear ubiquitin chains by NEMO is important for NF-kappaB activation. Cell. 2009;136(6):1098-109. https://doi.org/10.1016/j.cell.2009.03.007.

30. Swatek KN, Komander D. Ubiquitin modifications. Cell Res. 2016;26(4):399-422. https://doi.org/10.1038/cr.2016.39.

31. Rottenberg $\mathrm{S}$, Jonkers J. MEK inhibition as a strategy for targeting residual breast cancer cells with low DUSP4 expression. Breast Cancer Res. 2012;14(6):324. https://doi.org/10.1186/bcr3327.

32. Hwang WL, Yang MH, Tsai ML, et al. SNAIL regulates interleukin-8 expression, stem cell-like activity, and tumorigenicity of human colorectal carcinoma cells. Gastroenterology. 2011;141(1):279-91. https://doi. org/10.1053/j.gastro.2011.04.008.

33. Vega S, Morales AV, Ocaña OH, Valdés F, Fabregat I, Nieto MA. Snail blocks the cell cycle and confers resistance to cell death. Genes Dev. 2004:18(10):1131-43. https://doi.org/10.1101/gad.294104.

34. Lan L, Han $\mathrm{H}$, Zuo H, et al. Upregulation of myosin Va by Snail is involved in cancer cell migration and metastasis. Int J Cancer. 2010;126(1):53-64. https://doi.org/10.1002/ijc.24641.

\section{Publisher's Note}

Springer Nature remains neutral with regard to jurisdictional claims in published maps and institutional affiliations.
Ready to submit your research? Choose BMC and benefit from:

- fast, convenient online submission

- thorough peer review by experienced researchers in your field

- rapid publication on acceptance

- support for research data, including large and complex data types

- gold Open Access which fosters wider collaboration and increased citations

- maximum visibility for your research: over $100 \mathrm{M}$ website views per year

At BMC, research is always in progress.

Learn more biomedcentral.com/submissions 